\title{
Predictors of long-term prognosis in rheumatoid arthritis-related interstitial lung disease
}

Juan Chen ( $\nabla$ juchne@xmu.edu.cn )

Xiamen University and Fujian Medical University Affiliated First Hospital

\section{Yaqiong Chen}

Xiamen University and Fujian Medical University Affiliated First Hospital

\section{Dehao Liu}

Xiamen University and Fujian Medical University Affiliated First Hospital

Yihua Lin

Xiamen University and Fujian Medical University Affiliated First Hospital

\section{Lei Zhu}

University of Pittsburgh School of Medicine

\section{Shuli Song}

Xiamen University and Fujian Medical University Affiliated First Hospital

\section{Yudi Hu}

Medical College of Xiamen University

\section{Tao Liang}

Medical College of Xiamen University

\section{Yongliang Liu}

Xiamen University School of Public Health

Wei Liu

Xiamen University School of Public Health

\section{Lin Weng}

Xiamen University and Fujian Medical University Affiliated First Hospital

\section{Qiyuan Li}

Medical College of Xiamen University

\section{Shengxiang Ge}

Xiamen University School of Public Health

\section{Dana P. Ascherman}

University of Pittsburgh School of Medicine

\section{Research Article}


Keywords: interstitial lung disease (ILD), rheumatoid arthritis (RA), prognosis, biomarkers, longitudinal outcome

Posted Date: October 26th, 2021

DOl: https://doi.org/10.21203/rs.3.rs-707140/v2

License: (c) (i) This work is licensed under a Creative Commons Attribution 4.0 International License. Read Full License 


\section{Predictors of long-term prognosis in rheumatoid arthritis-related interstitial}

\section{lung disease}

Short running head: Predictors of RA-ILD prognosis

Juan Chen ${ }^{1 \S *}$, Yaqiong Chen ${ }^{1 *}$, Dehao $\mathrm{Liu}^{2}$,Yihua $\mathrm{Lin}^{3}$, Lei Zhu ${ }^{4}$, Shuli Song ${ }^{1}$, Yudi $\mathrm{Hu}^{5}$, Tao Liang ${ }^{5}$, Yongliang Liu ${ }^{6}$, Wei Liu ${ }^{6}$, Lin Weng ${ }^{1}$, Qiyuan $\mathrm{Li}^{5}$, Shengxiang $\mathrm{Ge}^{6}$, Dana P. Ascherman ${ }^{4 \S}$

1Division of Rheumatology and Clinical Immunology, Xiamen University and Fujian Medical University Affiliated First Hospital, Xiamen, Fujian, China

${ }^{2}$ Division of Radiology, Xiamen University and Fujian Medical University Affiliated First Hospital, Xiamen, Fujian, China

${ }^{3}$ Division of Respiratory Medicine, Xiamen University and Fujian Medical University Affiliated First Hospital, Xiamen, Fujian, China

${ }^{4}$ Division of Rheumatology and Clinical Immunology, University of Pittsburgh

School of Medicine, Pittsburgh, USA

${ }^{5}$ Department of Translational Medicine, Center for Bio Medical Big Data Research,

Medical College of Xiamen University, Xiamen, Fujian, China

${ }^{6}$ State Key Laboratory of Molecular Vaccinology and Molecular Diagnostics, Xiamen University School of Public Health, Xiamen, Fujian, China

*Dr. Juan Chen and Dr.Yaqiong Chen contributed equally to this paper. 
All authors declare no conflicts of interest.

\section{$\S_{\text {Corresponding authors }}$}

Dr. Dana P. Ascherman,

Division of Rheumatology and Clinical Immunology,

University of Pittsburgh School of Medicine,

Pittsburgh, 15261

USA

Email: DASCHER@pitt.edu

ORCiD iD: 0000-0002-1387-0860

Juan Chen

Rheumatology Department, First Hospital of Xiamen University

ZhenHai Road No.55, Xiamen, China

Tel: (86) 5922137609

Email: juchne@xmu.edu.cn

ORCiD iD: 0000-0002-3328-9627

\section{Funding}

This study was funded by the National Natural Science Foundation of China (Grant 
No. 81771751) to Dr. Juan Chen and Fujian Provincial Department of Science and Technology (Grant No. 2018J01383) to Dr. Juan Chen.

Key Indexing Terms: interstitial lung disease (ILD), rheumatoid arthritis (RA), prognosis, biomarkers, longitudinal outcome 


\begin{abstract}
The aim of the study was to identify specific clinical and serum protein biomarkers that are associated with longitudinal outcome of RA-associated interstitial lung disease (RA-ILD). 60 RA patients with clinical and serological profiles were assessed by HRCT and pulmonary function tests (PFTs) at baseline (Year 0) and 5 years post enrollment (Year 5). Progression versus non-progression was defined based on changes in Quantitative Modified HRCT scores and PFTs over time. Specific serum protein biomarkers were assessed in serum samples at baseline and Year 5 by Multiplex enzyme-linked immunosorbent assays (ELISAs).
\end{abstract}

At Year 5, 32\% of patients demonstrated progressive RA-ILD, 35\% were stable, and $33 \%$ improved. Baseline age and rheumatoid factor (RF) were significantly different between RA-ILD outcomes of progression vs. no-progression $(p<0.05)$. Changes in levels of CXCL11/I-TAC and MMP13 over 5 years also distinguished pulmonary outcomes $(p<0.05)$. A final binary logistic regression model revealed that baseline age and changes in serum MMP13 were associated with RA-ILD progression at Year $5(p<0.05)$, with an AUC of 0.7569.

Collectively, these analyses demonstrated that baseline clinical variables (age, RF) and shifts in levels of selected serum proteins (CXCL11/I-TAC, MMP13) were strongly linked to RA-ILD outcome over time. 


\section{Introduction}

Rheumatoid arthritis (RA) may cause a variety of extra-articular manifestations,

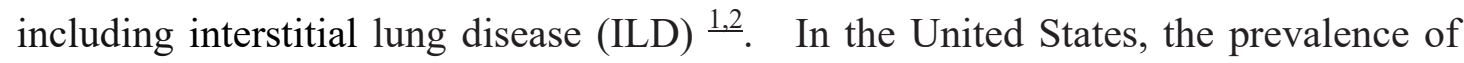
RA-ILD ranged from 3.2 to 6.0 cases per 100,000 people from 2003 to $2014^{\underline{3}}$. Equally important, a number of studies have indicated that RA-ILD causes decreased

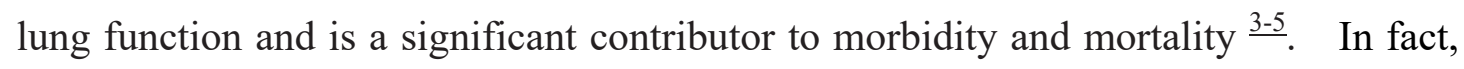
pulmonary complications are directly responsible for $10-20 \%$ of mortality in RA, corresponding to an estimated standardized mortality ratio of 2.5-5.0 compared with control populations $\underline{6}$. Within 5 years of index date, about one-third of RA-ILD patients with a potential linkage to Social Security Administration Death Index (SSDI) died ${ }^{3}$. In another study, the 5-year mortality was $39.0 \%$ versus $18.2 \%$ with and without ILD ${ }^{7}$.

Given the previously discussed morbidity/mortality of RA-ILD, it is necessary to find methods to not only make an early diagnosis, but also to reliably predict disease progression - particularly in individuals with subclinical and/or radiographically mild disease. Identifying baseline characteristics and longitudinal markers of disease progression would improve prognostication and help guide management of these patients. Demonstrating the feasibility of this approach, previous cross-sectional studies have identified a number of serum protein biomarkers that are associated with the presence of ILD in RA patients, many of which are also associated with idiopathic 
pulmonary fibrosis (IPF $)^{8-12}$. However, the ability of these and other biomarkers that focus on biological pathways involved in RA-ILD pathogenesis to predict disease

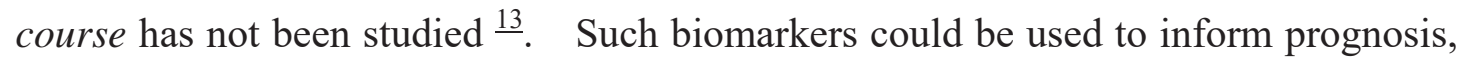
suggest potential treatment options, and identify new areas of focus for drug discovery $\frac{13}{}$. In this study, we therefore sought to identify specific clinical and proteomic biomarkers associated with longitudinal outcomes in RA-ILD.

\section{Results}

\section{Baseline demographic and radiographic characteristics of the longitudinal} cohort.

A total of 60 RA patients were recruited in 2013, and we followed them longitudinally for the 5-year period of this study. The cohort consisted of predominantly female $(80 \%)$ never-smokers $(86.7 \%)$, and their baseline mean age was $47.6 \pm 16.6$ years (Table1). $\quad 4 / 60$ patients $(6.7 \%)$ died due to progressive ILD and/or pneumonia, resulting in a 5-year survival rate of 93\% (Figure 1), with radiographic evidence of advanced RA-ILD (UIP pattern) at baseline associated with a DLco ranging from $50-60 \%$ predicted.

At baseline, these 60 patients had varying degrees of parenchymal lung abnormalities and were subclassified as RA-no ILD (ILA=0), RA-indeterminate ILD (ILA=1), RA-mild ILD (ILA=2), and RA-moderate/severe ILD (ILA=3) (Table 1). As shown 
in Table 1, Quantitative Modified ILD scores corresponding to subcategories of indeterminate, mild, and advanced ILD were differed significantly from those associated with RA-no ILD ( $p<0.001$, respectively). Review of Table 1 also indicates that among baseline clinical and demographic variables, age was differed significantly between ILA subgroups.

\section{Relationship between baseline ILD-specific variables and longitudinal} outcomes.

Baseline HRCT. Changes in HRCT based on 0-3 ILA scores from Year 0 to Year 5 were analyzed, demonstrating an increase in the number of RA-no ILD (ILA 0) patients from $22(37 \%)$ to $25(42 \%)$, a decrease in patients with indeterminate RA-ILD (ILA 1) from $23(38 \%)$ to 21 (35\%), and a decrease in number of patients with mild RA-ILD (ILA 2) from 10 (17\%) to 9 (15\%). However, the number of patients with advanced RA-ILD (ILA 3) did not change and remained at 5 (8\%) (data not shown).

When longitudinal outcome was evaluated by Quantitative Modified ILD scoring, 19 patients (including the 4 patients who died) (32\%) became worse, 21 patients $(35 \%)$ remained unchanged/stable, and 20 patients (33\%) improved (Figure.1). Comparative statistical analysis indicates that the Quantitative Modified ILD scoring 
system was more sensitive to changes in radiographic status that did not necessarily result in a shift in ILA subcategory. Despite this enhanced sensitivity for change in radiographic severity, baseline Quantitative Modified ILD scores were only slightly higher in progressors versus non-progressors (2.0 versus $1.5, p>0.05)$ and therefore not predictive of worsening RA-ILD (Table 2).

To further explore the relationship between baseline HRCT characteristics and risk of disease progression, we assessed the correlation between patterns of HRCT abnormalities at baseline and disease outcome. While $26 \%$ of patients $(n=5)$ in the progression group had evidence of UIP at baseline (versus 74\% non-UIP, $n=14$ ), none of the patients in the no-progression group had UIP at baseline (Table 2).

PFTs. The associations between baseline pulmonary function test parameters and outcomes determined by Quantitative Modified ILD scores in HRCT were analyzed (Table 2). Baseline percent predicted DLCO (DLco\%) in the progression group was significantly lower than in the no-progression group $(p<0.01)$. However, no significant differences in baseline percent predicted FVC (FVC\%) or FEV1 (FEV1\%) separated the progression versus no-progression groups $(p>0.05)$ (Table 2). Among the 56 patients who were alive at Year 5, 39 had available PFT data from both Year 0 and Year 5 time points. While 7 patients (18\%) became worse based on defined FVC criteria, 19 (49\%) remained unchanged/stable, and 13 (33\%) improved (data not 
shown). The overall correlation between changes of FVC and HRCT severity scores was $0.84(p<0.05)$.

\section{Baseline clinical risk factors, medication usage, and longitudinal outcomes.}

As shown in Table 2, the relationships between baseline clinical parameters (age, sex, smoking history, RA disease duration, articular disease activity (DAS28), RF, and anti-CCP) and long-term outcomes based on changes of the Quantitative Modified ILD HRCT scores over five years were analyzed. Baseline age in the progression group was significantly higher than baseline age in the no-progression group $(p<0.01)$. Similarly, baseline titers of RF in the progression group were significantly higher than baseline titers of RF in the no-progression group $(p<0.05)$ (Table 2). Of note, pack-years of smoking in the progression group were higher $(3.7 \pm 9.0)$ than in the no-progression group $(1.6 \pm 7.3 ; p>0.05)$. However, baseline sex, anti-CCP antibodies, and articular disease activity (measured by DAS28) were not different between the progression and no-progression groups $(p>0.05)$. Finally, four patients (6.7\%) with symptoms of cough and/or dyspnea were in the progression outcome group, while none of the patients in the no progression group had these symptoms - a difference that was statistically significant $(p<0.01$, Table 2$)$.

Medications used. There was a statistically significant association between cumulative dosages of methotrexate (MTX) used and outcome measured by the 
Quantitative Modified ILD HRCT scores, as the cumulative dosage of methotrexate used in the progression group was significantly lower than that in the no-progression group $(p<0.05)$ (Table 2). In contrast, there were no significant associations between cumulative dosages of leflunomide or prednisone and ILD outcomes (Table 2).

\section{Serum levels of biomarkers and longitudinal outcomes.}

To determine serum biomarkers that might be associated with the long-term prognosis of RA-ILD, we measured serum levels of 36 proteins consisting of cytokines, chemokines, growth factors, and remodeling proteins (MMPs) that have previously

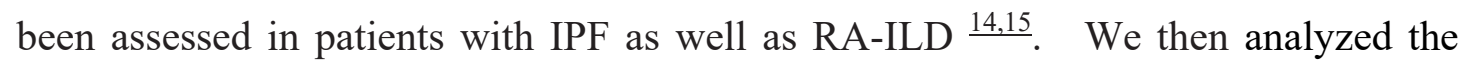
relationship between serum levels of these serum biomarkers (both baseline and change over time) and outcomes. As shown in Supplementary Figure 2 and 3, none of the assessed protein levels (at baseline) were associated with RA-ILD outcome (progression versus no-progression) measured by shifts in the $\mathrm{FVC} \%$, DLco\%, or Quantitative Modified ILD HRCT scores from Year 0 to Year $5(p>0.05)$.

As a complementary approach to identify markers of RA-ILD progression, we assessed the correlation between changes in serum levels of selected cytokines/chemokines/MMPs and RA-ILD outcomes measured by PFT parameters as well as Quantitative Modified ILD HRCT scores. As shown in Figure 2, changes in 
serum levels of CXCL11 and MMP-13 from Year 0 to Year $5(\log$ Year 5 - $\log$ Year 0) were significantly associated with different outcomes (progression versus no-progression) measured by the Quantitative Modified HRCT scores $(p<0.05)$. However, levels of these serum proteins were not statistically associated with different outcomes measured by PFT parameters. Correlations between shifts in the serum levels of other defined protein biomarkers and radiographic/PFT outcomes between Year 0 and Year 5 were not statistically significant and are reported in Supplementary Figure 4.

\section{Factors predicting progression of RA-ILD}

More detailed statistical analyses were conducted to see which combination of demographic variables, clinical risk factors (RF, anti-CCP, DSA28 and disease duration at baseline), and serum proteins (CXCL9/MIG, CXCL10/IP10, CXCL11/I-TAC and MMP13 over 5-year) were associated with binary outcomes of progression versus no-progression. Significant differences were found between the progression and the no-progression groups in age, RF, and changes (log Year 5 - log Year 0) in serum levels of CXCL11/I- and MMP13 ( $p<0.05$, Table 3). We used the Spearman coefficient for continuous variables, and then one member of each pair of correlated variables $(r>0.3, p<0.05)$ was selected to include in the model to avoid multicollinearity. Age and change in MMP13 levels were the two variables that were selected to keep in the final logistic regression model to assess their association with 
disease outcome $(p<0.05$, Table 3$)$. Area under the curve derived from ROC analysis was 0.76 (95\% confidence interval: $0.62-0.90, p<0.05)$, indicating that the model incorporating age and change in MMP13 levels effectively distinguished disease progressors from non-progressors (Figure 3).

\section{Discussion}

This study demonstrated that several clinical/demographic variables and serum protein biomarkers were associated with the long-term prognosis of RA-ILD. While changes in serum levels of CXCL11 and MMP-13 were significantly associated with longitudinal progression of RA-ILD in univariate analyses, baseline age and RF were also associated with the long-term outcomes of RA-ILD. Our final binary logistic regression model revealed that baseline age and changes in serum levels of MMP13 were associated with RA-ILD progression (Yes. vs. No) at Year 5.

Of note, radiographic disease progression was accompanied by increased levels of CXCL11/I-TAC and MMP13 over this time period, indicating that these serum proteins could serve as prognostic biomarkers in our longitudinal cohort. However, the basis for these associations remains unclear. Furthermore, it is unclear why some biomarkers relate to disease activity at a specific time point, while other markers predict disease progression. Previous cross-sectional studies have shown, for 
example, that a number of serum protein biomarkers are associated with the presence of both mild and advanced forms of RA-ILD_-including MMP-7 and IP-10/CXCL10 9.16 — suggesting that these mediators contribute to the pathophysiology of RA-ILD. Moreover, a 7-biomarker signature consisting of MMP-1, MMP-2, MMP-7, MMP-9, IL-1 receptor antagonist, soluble CD40L, and CXCL9 effectively differentiated RA-ILD from RA-no ILD with high sensitivity and specificity in a separate cohort, yielding an AUC of $0.93^{\underline{8}}$.

We have extended these findings by showing that CXCR3-binding chemokines such as I-TAC/CXCL11 predict radiographic progression of RA-ILD, suggesting that pro-inflammatory disease pathways and TH1 skewing promoted by this chemokine contribute to progression of ILD. On the other hand, the demonstrated relationship between shifting MMP13 levels and disease outcome points to an important role for tissue remodeling in progression of RA-ILD.

Our present study indicated that older age was a clinical risk factor associated with worse long-term RA-ILD outcomes, correlating positively with the risk of progressive RA-ILD. These findings dovetail with other studies demonstrating that older age

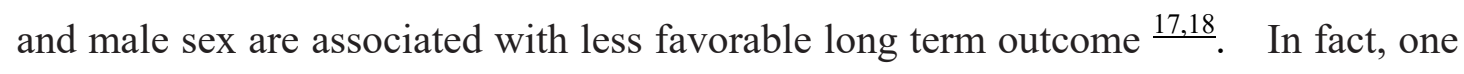


study has shown that for every 10 year increase in age, the risk of ILD increased by $59.9 \%$ in $\mathrm{RA}^{19}$.

In addition to age, baseline RF titters were also associated with RA-ILD outcome in our study — though we did not observe similar associations with baseline levels of anti-CCP antibodies. Although the literature does support some correlation between the levels of anti-CCP/anti-CCP2 antibodies and the likelihood of RA-ILD $\stackrel{8,20,21}{\text {, our }}$ failure to see any statistical correlation with disease outcome could reflect the absence of lung-specific antigens in CCP ELISAs.

Apart from these biomarker analyses, a novel observation emerging from our study was that the prognosis of indeterminate/mild RA-ILD was relatively favorable over the 5 year period of this study. In fact, 33\% of RA-ILD patients in these subcategories improved over this time period based on HRCT criteria, and 33\% of patients improved according to changes of FVC. One plausible explanation for the discrepancy between our findings and previous studies demonstrating much worse

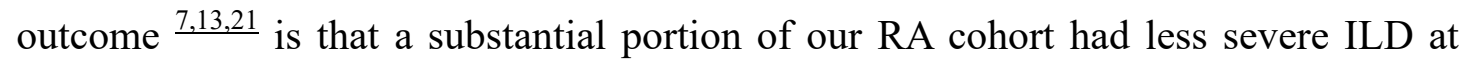
study entry (37\% no-ILD and 38\% indeterminate ILD). Comparing baseline ILD scoring and outcome in our study demonstrated that more individuals with baseline ILA scores of 0,1 , and 2 were in the no-progression group (41/56 individuals with ILA 0-2 did not progress) relative to those individuals with a baseline ILA score of 3 (where all 5 patients progressed). Therefore, RA patients with less severe stages of 
ILD (e.g., indeterminate/mild ILD) at baseline are less likely to progress - though our data do not allow us to conclude whether this trend reflects the natural history of mild/subclinical ILD or if these milder forms of RA-ILD are more responsive to immunomodulatory treatments targeting articular disease.

Beyond these considerations, another reason for generally favorable outcomes in this cohort could be that most of enrolled patients $(80 \%)$ were female with very low primary smoking rates. In support of these hypotheses, our data show that the percentage of female RA-ILD patients was higher among non-progressors (85\%) than in progressors $(68 \%)$. On the other hand, pack-years of smoking in the progression group were higher $(3.7 \pm 9.0$ pack-years $)$ than in the no-progression group $(1.6 \pm 7.3$ pack-years; $p>0.05$ ). The latter observation is consistent with previous studies indicating that cigarette smoking is a risk factor and an independent predictor of RA-ILD $\stackrel{8,22-24}{\text {. }}$

In terms of baseline imaging characteristics, all 41 patients in the no-progression outcome group had HRCTs showing a non-UIP pattern with predominant bilateral, ground-glass opacities--contrasting with the progression group in which only $74 \%$ of patients demonstrated a non-UIP pattern. Distinguishing UIP from non-UIP patterns

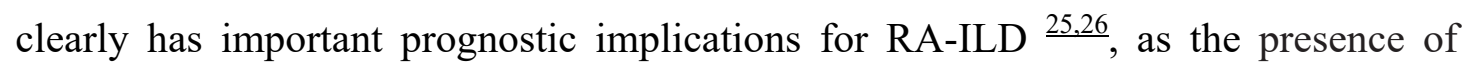


radiological honeycombing may be a useful predictor of poor prognosis $\frac{17,26,27}{\text {. In }}$ fact, UIP-patterned ILD resembles idiopathic pulmonary fibrosis (IPF) and was the

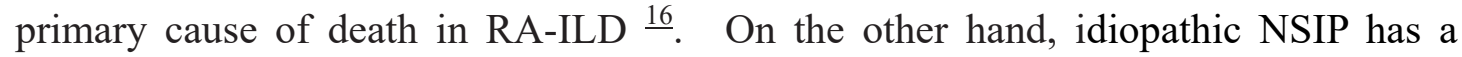
better prognosis and often responds to anti-inflammatory therapy ${ }^{25}$, suggesting that inflammatory cells likely to be present in non-UIP forms of RA-ILD might be responsive to immunosuppressive treatment $\frac{28}{}$. In turn, because changes in the therapeutic approach to RA patients with ILD may alter the profile and outcome of this disease $\mathrm{e}^{27}$, detection and management of RA-ILD patients at earlier stages with non-UIP HRCT patterns could prevent progression to more advanced stages of this devastating extra-articular disease complication.

Our study has several limitations. Importantly, this study was a single-center study with a relatively small number of patients in each subgroup of RA-ILD having follow-up data. Beyond these issues, not every subject included in this study had PFT data available. Given these and other limitations, longitudinal assessment of additional RA-ILD patients from larger, independent cohorts will be necessary to verify our findings and fully determine the predictive value of identified biomarkers, including their ability to distinguish outcomes of RA-ILD from those of other forms of ILD and/or IPF. 


\section{Conclusions}

While baseline age and RF predicted RA-ILD outcomes of progression versus no-progression, changed levels of CXCL11/I-TAC and MMP-13 over 5 years were also correlated with long-term prognosis of RA-ILD. In multivariate analyses, baseline age and changed levels of MMP-13 were associated with the risk of progression of RA-ILD.

\section{Methods}

\section{Patients.}

The study was designed as a 5-year, single-center, observational study that was conducted at the Division of Rheumatology, Xiamen University Affiliated First Hospital, China. Ethical approval for the study was obtained by the Ethical Committee of Xiamen University Affiliated First Hospital (Approval number.KY2017-026). All experiments were performed in accordance with relevant guidelines and regulations.

60 adult patients with RA ( $>18$ years old) who met the American College of

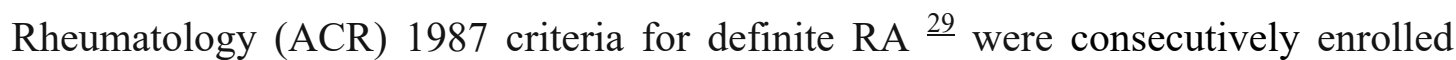
between July 2012 and March $2013^{9}$ and followed for a minimum of 5 years. Written informed consent was obtained from each participant. Serum samples were 
collected at baseline (Year 0) and at 5-year study visits (Year 5). Clinical data were obtained within 3 days of serum sampling.

RA patient-specific variables consisting of baseline age, sex, disease duration, smoking history (never/current/former smoking as well as second-hand cigarette smoke exposure), articular disease activity (DAS28), titers of RF, and anti-CCP were recorded (Supplementary Figure 1). Dyspnea was evaluated with the University of

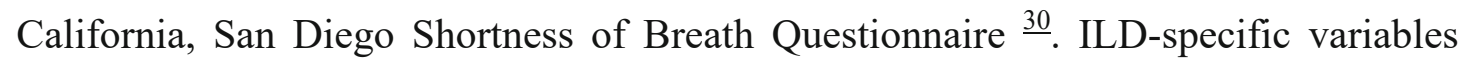
including high-resolution computed tomography scans (HRCT) and pulmonary function tests (PFTs) [forced vital capacity percent (FVC\%), forced expiratory volume in 1 second (FEV1\%) and lung diffusion for carbon monoxide (DLco\%)] were measured. Selected biomarker variables, which included a range of cytokines, chemokines, acute-phase proteins, and MMPs potentially related to the mechanism of RA-ILD, were tested. All clinical, radiographic, PFT, and biomarker indices were collected at baseline and Year 5. Medical history and the use of disease-modifying anti-rheumatic drugs (DMARDs) and/or corticosteroids were obtained at these time points.

\section{Imaging and scoring.}


HRCT images were obtained from all patients at Year 0 and Year 5. A numerical score was assigned based on the type and distribution of interstitial lung abnormalities (ILA) consisting of septal lines, reticulation, traction bronchiectasis, cyst formation, and/or ground-glass attenuation, utilizing a published scale of ILA ranging from 0-3 9,31,32. In terms of RA-ILD sub-classification, predominant bilateral, ground-glass opacities without honeycombing or architectural distortion were designated as a non-UIP pattern, while predominant honeycombing and traction bronchiectasis or architectural distortion were identified as a UIP pattern.

When Year 0 and Year 5 HRCT images from the same patient were compared, ILD severity was calculated using a more detailed Quantitative Modified ILD scoring system in which each lung was divided into three zones of upper, middle and lower at the level of the aortic arch and the left inferior pulmonary vein. In the Quantitative Modified ILD scoring system, the following HRCT findings were coded as present or absent in each zone: ground-glass opacity (GGO), consolidation /air space disease (AS), mixed GGO/AS, parenchymal micronodules $(<7 \mathrm{~mm})$, reticular opacities, linear opacities or septal lines, peri-bronchovascular thickening, honeycombing, and traction bronchiectasis/bronchiolectasis. The severity of abnormalities in each of the defined lung zones was then graded as: $0=$ no abnormality; $1=$ trace/minor abnormality $(<5 \%$ area); $2=$ mild abnormality $(5-20 \%$ area $) ; 3=$ moderate or significant abnormality 
$(>20-50 \%$ area); or $4=$ severe abnormality ( $>50 \%)$ (Supplementary Table 1$)$. The maximum possible ILD score was 192 in this grading scheme.

Quantitative Modified ILD scores at Year 0 and at Year 5 for each patient were assessed by three independent reviewers who were blinded with regard to the clinical status of patients. Scans with discrepant readings were then evaluated and resolved by consensus.

\section{RA-ILD disease outcome at Year 5 based on HRCT and PFTs.}

RA-ILD radiographic status at Year 5 was defined as unchanged/stable, improved, or worse based on changes in the Quantitative Modified ILD HRCT score relative to ILD scores at Year 0. Changes in ILD score between $0-10 \%$ at Year 0 and Year 5 were classified as unchanged/stable, while reductions in ILD scores by greater than $10 \%$ were classified as improved (score reduction $>10 \%$ ). Conversely, increases in ILD scores by more than $10 \%$ were classified as worse (score increase $>10 \%$ ).

ILD disease outcome was also measured based on relative changes in functional parameters (FVC and DLco) between Year 0 and Year 5. While greater than $10 \%$ decreases in the ratio of Year 5/Year $0 \mathrm{FVC}$ and/or DLco were defined as worse, greater than $10 \%$ increases in the ratio of Year 5/Year $0 \mathrm{FVC}$ and/or DLco were 
classified as improved and less than $10 \%$ change in the Year 5/Year 0 FVC and/or DLco were considered unchanged/stable $\stackrel{33,34}{\text {. }}$.

\section{Multiplex ELISA.}

Multiplex ELISAs were performed using Luminex xMAP technology in a 96-well microplate format (eBioscience, Procarta). A combined 36-plex assay was used to determine serum levels of a range of cytokines, chemokines, acute-phase proteins, and MMPs (listed in Supplementary Table 2) potentially related to the mechanism of RA-ILD. Of note, serum samples at Year 0 and at Year 5 were assessed at the same time for all enrolled patients.

\section{Statistical Analysis.}

All continuous variables were evaluated for normality to ensure uniformity in concentration and dispersion. Logarithm transformed values were used for variables not adhering to a normal distribution. While mean and standard deviation were presented for normally distributed variables, median and interquartile range (IQR) were presented for variables that did not follow a normal distribution. 
Bivariate analyses were conducted using Chi-square or Fisher's exact test for categorical variables and outcomes; alternatively, T tests or Mann-Whitney U tests were used for continuous variables (e.g., serum biomarker levels) and outcomes, and comparing the characteristics of different groups of RA-ILD patients at baseline. To determine correlations among continuous variables and analyze the influence of confounding factors, Spearman correlation coefficients were calculated.

Additional analyses were conducted to see which demographic variables, clinical risk factors, or serum proteins were associated with disease progression. These variables were included in the final logistic regression model as candidate covariates to assess associations with the outcome of disease progression versus no progression. We further evaluated the performance of our classification model using receiver operating characteristic (ROC) analysis to calculate area under the curve (AUC) values.

All statistical analyses were performed using IBM SPSS Statistics, version 20.0. Two-sided $p$-values $\leq 0.05$ were considered statistically significant. 


\section{Reference}

1 Castelino, F. V. \& Varga, J. Interstitial lung disease in connective tissue diseases: Evolving concepts of pathogenesis and management. Arthritis Res Ther. 12, 213 https://doi.org/10.1186/ar3097 (2010).

2 Nannini, C. et al. Incidence and mortality of obstructive lung disease in rheumatoid arthritis: A population-based study. Arthritis care \& research. 65, 1243-1250 https://doi.org/10.1002/acr.21986 (2013).

3 Raimundo, K. et al. Rheumatoid arthritis-interstitial lung disease in the united states: Prevalence, incidence, and healthcare costs and mortality. J Rheumatol. 46, 360-369 https://doi.org/10.3899/jrheum.171315 (2019).

4 Marigliano, B., Soriano, A., Margiotta, D., Vadacca, M. \& Afeltra, A. Lung involvement in connective tissue diseases: A comprehensive review and a focus on $\begin{array}{lllll}\text { rheumatoid arthritis. Autoimmun } & \text { Rev. 12, }\end{array}$ https://doi.org/10.1016/j.autrev.2013.05.001 (2013).

5 Cavagna, L. et al. The multifaceted aspects of interstitial lung disease in rheumatoid arthritis. Biomed Res Int. 2013, 759760 https://doi.org/10.1155/2013/759760 (2013).

6 Brown, K. K. Rheumatoid lung disease. Proc Am Thorac Soc. 4, 443-448 https://doi.org/4/5/443 [pii]10.1513/pats.200703-045MS (2007).

7 Hyldgaard, C. et al. A population-based cohort study of rheumatoid arthritis-associated interstitial lung disease: Comorbidity and mortality. Annals of the $\begin{array}{lll}\text { rheumatic } & \text { diseases. } & \mathbf{7 6},\end{array}$ https://doi.org/10.1136/annrheumdis-2017-211138 (2017).

8 Kass, D. J. et al. Comparative profiling of serum protein biomarkers in rheumatoid arthritis-associated interstitial lung disease and idiopathic pulmonary fibrosis. Arthritis Rheumatol. 72, 409-419 https://doi.org/10.1002/art.41123 (2020).

9 Chen, J. et al. Biomarkers of rheumatoid arthritis-associated interstitial lung disease. Arthritis Rheumatol. 67, 28-38 https://doi.org/10.1002/art.38904 (2015).

10 Doyle, T. J. et al. Detection of rheumatoid arthritis-interstitial lung disease is enhanced by serum biomarkers. Am J Respir Crit Care Med. 191, 1403-1412 https://doi.org/10.1164/rccm.201411-1950OC (2015).

11 Amigues, I., Ramadurai, D. \& Swigris, J. J. Current perspectives on emerging biomarkers for rheumatoid arthritis-associated interstitial lung disease. Open Access Rheumatol. 11, 229-235 https://doi.org/10.2147/OARRR.S166070 (2019).

12 Lee, J. S. et al. Serum kl-6 levels reflect the severity of interstitial lung disease associated with connective tissue disease. Arthritis Res Ther. 21, 58 https://doi.org/10.1186/s13075-019-1835-9 (2019).

13 Assayag, D. et al. Predictors of mortality in rheumatoid arthritis-related interstitial lung disease. Respirology. 19, 493-500 https://doi.org/10.1111/resp.12234 (2014).

14 Rosas, I. O. et al. Mmp1 and mmp7 as potential peripheral blood biomarkers in idiopathic pulmonary fibrosis. Plos Med. 5, 623-633 https://doi.org/ARTN e9310.1371/journal.pmed.0050093 (2008). 
15 Richards, T. J. et al. Characterization and peripheral blood biomarker assessment of anti-jo-1 antibody-positive interstitial lung disease. Arthritis Rheum-Us. 60, 2183-2192 https://doi.org/10.1002/art.24631 (2009).

16 Yamakawa, H. et al. Impact of radiological honeycombing in rheumatoid arthritis-associated interstitial lung disease. BMC Pulm Med. 20, 25 https://doi.org/10.1186/s12890-020-1061-x (2020).

17 Bongartz, T. et al. Incidence and mortality of interstitial lung disease in rheumatoid arthritis: A population-based study. Arthritis Rheum. 62, 1583-1591 https://doi.org/10.1002/art.27405 (2010).

18 Koduri, G. et al. Interstitial lung disease has a poor prognosis in rheumatoid arthritis: Results from an inception cohort. Rheumatology (Oxford). 49, 1483-1489 https://doi.org/10.1093/rheumatology/keq035 (2010).

19 Zhang, Y., Li, H., Wu, N., Dong, X. \& Zheng, Y. Retrospective study of the clinical characteristics and risk factors of rheumatoid arthritis-associated interstitial lung disease. Clin Rheumatol. 36, 817-823 https://doi.org/10.1007/s10067-017-3561-5 (2017).

20 Alexiou, I. et al. Anti-cyclic citrullinated peptide-2 (cсp2) autoantibodies and extra-articular manifestations in greek patients with rheumatoid arthritis. Clin Rheumatol. 27, 511-513 https://doi.org/10.1007/s10067-007-0800-1 (2008).

21 Kelly, C. A. et al. Rheumatoid arthritis-related interstitial lung disease: Associations, prognostic factors and physiological and radiological characteristics--a large multicentre uk study. Rheumatology (Oxford). 53, 1676-1682 https://doi.org/10.1093/rheumatology/keu165 (2014).

22 Hwang, J. Y., Randall, T. D. \& Silva-Sanchez, A. Inducible bronchus-associated lymphoid tissue: Taming inflammation in the lung. Front Immunol. 7, 258 https://doi.org/10.3389/fimmu.2016.00258 (2016).

23 Ascherman, D. P. Interstitial lung disease in rheumatoid arthritis. Curr Rheumatol Rep. 12, 363-369 https://doi.org/10.1007/s11926-010-0116-z (2010).

24 Darrah, E. \& Andrade, F. Rheumatoid arthritis and citrullination. Curr Opin Rheumatol. 30, $72-78$ https://doi.org/10.1097/BOR.0000000000000452 (2018).

25 Kim, E. J., Collard, H. R. \& King, T. E., Jr. Rheumatoid arthritis-associated interstitial lung disease: The relevance of histopathologic and radiographic pattern. Chest. 136, 1397-1405 https://doi.org/10.1378/chest.09-0444 (2009).

26 Yamakawa, H. et al. Predictive factors of mortality in rheumatoid arthritis-associated interstitial lung disease analysed by modified hrct classification of idiopathic pulmonary fibrosis according to the 2018 ats/ers/jrs/alat criteria. J Thorac Dis. 11, 5247-5257 https://doi.org/10.21037/jtd.2019.11.73 (2019).

27 Giles, J. T. et al. Association of cross-reactive antibodies targeting peptidyl-arginine deiminase 3 and 4 with rheumatoid arthritis-associated interstitial lung disease. PLoS One. 9, e98794 https://doi.org/10.1371/journal.pone.0098794 (2014).

28 Volkmann, E. R. et al. Progression of interstitial lung disease in systemic sclerosis: The importance of pneumoproteins krebs von den lungen 6 and ccl18. Arthritis Rheumatol. 71, 2059-2067 https://doi.org/10.1002/art.41020 (2019). 
29 Arnett, F. C. et al. The american rheumatism association 1987 revised criteria for the classification of rheumatoid arthritis. Arthritis Rheum. 31, 315-324 https://doi.org/10.1002/art.1780310302 (1988).

30 Eakin, E. G., Resnikoff, P. M., Prewitt, L. M., Ries, A. L. \& Kaplan, R. M. Validation of a new dyspnea measure: The ucsd shortness of breath questionnaire. University of california, san diego. Chest. 113, 619-624 (1998).

31 Washko, G. R. et al. Lung volumes and emphysema in smokers with interstitial lung abnormalities. N Engl J Med. 364, 897-906 https://doi.org/10.1056/NEJMoa1007285 (2011).

32 Washko, G. R. et al. Identification of early interstitial lung disease in smokers from the copdgene study. Acad Radiol. $17, \quad 48-53$ https://doi.org/10.1016/j.acra.2009.07.016 (2010).

33 Flaherty, K. R. et al. Design of the pf-ild trial: A double-blind, randomised, placebo-controlled phase iii trial of nintedanib in patients with progressive fibrosing interstitial lung disease. BMJ Open Respir Res. 4, e000212 https://doi.org/10.1136/bmjresp-2017-000212 (2017).

34 Yamakawa, H. et al. Prognostic factors and disease behaviour of pathologically proven fibrotic non-specific interstitial pneumonia. Respirology. 23, 1032-1040 https://doi.org/10.1111/resp.13313 (2018).

\section{Acknowledgement}

We thank all patients who participated in the study cohort.

\section{Authors' contributions}

Study conception or design: J.C and D.A; acquisition of the data: D.H.L.,Y.H.L.,S.L.S.,Y.L.L., W.L., W.L., and S.X.G.; analysis and interpretation of the data: Q.Y.L.,Y.Q.C., D.A., L.Z., Y.D.H., and T.L.; drafting and revising the article: J.C., D.A., and L.Z. All authors read and approved the final manuscript.

\section{Online supplement}

Supplementary material accompanies the online version of this article.

\section{Competing interests}

The authors declare no conflicts of interests. 


\section{Figure Legends}

Figure 1. Study enrollment at Year 0 and Year 5 A flow diagram of the study shows the different degrees of RA-ILD based on 0-3 ILA scores in HRCT at Year 0 and outcome at Year 5 based on changes of Quantitative Modified ILD scores. Changes in the Quantitative Modified ILD score between $0-10 \%$ at Year 0 and Year 5 were classified as stable, while reduction of ILD scores by greater than $10 \%$ were classified as better (score reduction $>10 \%$ ) and changes more than $10 \%$ increased as worse. $\quad \mathrm{HRCT}=$ high-resolution computed tomography; ILD = interstitial lung disease; ILA= interstitial lung abnormality; RA=rheumatoid arthritis.

Figure 2. Relationship between changes in serum levels of biomarkers from Year 0 to Year 5 and radiographic outcome. Changes of serum levels of A) CXCL11/I-TAC and B) MMP-13 from Year 0 to Year 5 (log Year $5-\log$ Year 0) (y-axis) were significantly associated with RA-ILD outcomes (progression versus no-progression, $\mathrm{x}$-axis) assessed by changes in Quantitative Modified ILD scores ( $p=0.036, p=0.014$, respectively). Horizontal bars signify median values.

Figure 3. ROC analyses of the disease prediction models Age and changes in serum levels of MMP13（log Year 5-log Year 0) over 5-years were fit into the final logistic regression model. ROC analyses for the models: 1) with age; 2) with 
changes in MMP13 level; and 3) with the combination of age and changes in MMP13 level demonstrated the model with the combinations of the variables predicts the risk of progression in RA-ILD (as measured by Quantitative Modified ILD scores) the best. The respective AUCs for those models are 0.6756, 0.7138 and $0.7569(p=0.04$, 0.02 and $<0.01)$. 
Table 1 Characteristics of 60 RA patients at baseline

\begin{tabular}{|c|c|c|c|c|}
\hline & $\begin{array}{c}\text { RA-no ILD } \\
(\text { ILA score 0) } \\
(\mathbf{n}=\mathbf{2 2})\end{array}$ & $\begin{array}{c}\text { RA-indeterminate ILD } \\
\text { (ILA score 1) } \\
(\mathbf{n}=\mathbf{2 3})\end{array}$ & $\begin{array}{l}\text { RA-mild ILD } \\
\text { (ILA score 2) } \\
\quad(n=10)\end{array}$ & $\begin{array}{c}\text { RA-advanced ILD } \\
\text { (ILA score 3) } \\
(\mathbf{n}=\mathbf{5})\end{array}$ \\
\hline \multicolumn{5}{|l|}{ Demographic parameters } \\
\hline Age, years, mean $\pm \mathrm{SD}$ & $39.4 \pm 15.8$ & $51.2 \pm 15.4^{*}$ & $60.2 \pm 5.4^{* *}$ & $74.8 \pm 15.1^{* *}$ \\
\hline Female, no. $(\%)$ & $18(82)$ & $21(91)$ & $7(70)$ & $2(40)$ \\
\hline \multicolumn{5}{|l|}{ Smoker, no. $(\%)$} \\
\hline Never & $21(95)$ & $23(100)$ & $9(90)$ & $3(60)$ \\
\hline Former & $0(0)$ & $0(0)$ & $0(0)$ & $2(40)$ \\
\hline Current & $1(5)$ & $0(0)$ & $1(10)$ & $0(0)$ \\
\hline Pack-years of smoking, mean $\pm \mathrm{SD}$ & $1.8 \pm 8.5$ & $1.7 \pm 5.8$ & $3.0 \pm 9.5$ & $5.0 \pm 11.2$ \\
\hline Pack-years of secondhand smoking, mean \pm SD & $0 \pm 0$ & $1.1 \pm 3.7$ & $5.0 \pm 8.5$ & $8.0 \pm 17.9$ \\
\hline \multicolumn{5}{|l|}{ RA parameters, median (IQR) } \\
\hline $\mathrm{RF}, \mathrm{IU} / \mathrm{ml}$ & $45.0(20.1-152.0)$ & $183.5(60.5-232.8)$ & $226.0(45.1-569.0)$ & $300(64.1-673.5)$ \\
\hline Anti-CCP, units/ml & $86.9(48.4-298.5)$ & $328.0(59.4-470.0)$ & $480.2(87.2-500.0)$ & $301.0(173.2-500.0)$ \\
\hline DAS28 & $4.0(2.4-5.3)$ & $4.1(3.3-5.2)$ & $4.8(3.7-5.4)$ & $4.2(3.0-4.7)$ \\
\hline Duration of RA, years & $3.5(1.0-8.0)$ & $2.0(0.5-10)$ & $3.3(1.0-9.3)$ & $10.0(2.0-15.0)$ \\
\hline \multicolumn{5}{|l|}{ Medication use (ever) at ILD diagnosis, no. (\%) } \\
\hline Methotrexate & $22(100)$ & $23(100)$ & $10(100)$ & $5(100)$ \\
\hline Leflunomide & $10(45)$ & $9(39)$ & $3(30)$ & no use \\
\hline Coticosteroid & $2(9)$ & $4(17)$ & $2(20)$ & $2(40)$ \\
\hline Biologic & $2(9)$ & $1(4)$ & no use & no use \\
\hline \multicolumn{5}{|l|}{ Respiratory parameters, no. (\%) } \\
\hline Cough & 0 & 0 & 0 & $4(80) * *$ \\
\hline & & 28 & & \\
\hline
\end{tabular}


Dyspnea

0

0

0

$4(80) * *$

\begin{tabular}{|c|c|c|c|c|}
\hline \multicolumn{5}{|c|}{ Spirometric parameters, median (IQR) } \\
\hline FEV1, percent of predicted & $83.0(76.0-98.0)$ & $82.0(74.0-90.0)$ & $82.0(58.8-94.3)$ & $72.0(57.5-97.8)$ \\
\hline FVC, percent of predicted & $79.0(75.0-94.0)$ & $83.0(76.0-89.0)$ & $74.0(65.5-83.0)$ & $69.5(59.3-104.5)$ \\
\hline Dlco, percent of predicted & $86.0(75.0-105.0)$ & $94.0(68.0-101.5)$ & $65.0(59.0-70.0)^{*}$ & $52.5(51.0-61.5)^{* *}$ \\
\hline \multicolumn{5}{|c|}{$\begin{array}{l}\text { Quantitative Modified ILD scoring in HRCT, median } \\
\text { (IQR) }\end{array}$} \\
\hline & $0.8(0.0-1.0)$ & $2.0(1.0-4.0) * * *$ & $8.0(3.6-12.1)^{* * *}$ & $13.0(8.1-24.5)^{* * *}$ \\
\hline
\end{tabular}

$\mathrm{RF}=$ rheumatoid factor; anti-CCP $=$ anti-cyclic citrullinated peptide; DAS28 $=28$-joint Disease Activity Score. IQR $=$ interquartile range. Age was statistically significant

differences in different subgroups: RA-mild ILD (ILA score2) and RA-no ILD (ILA score 0) ( $p<0.05$ ), RA--advanced ILD (ILA score3) and RA-no-ILD (ILA score0)

$(p<0.01)$. The Quantitative Modified ILD scores in HRCT in RA-indeterminate ILD, RA-mild ILD and RA-advanced ILD groups were statistically significant different than

RA-no ILD $(p<0.001)$.

$* p<0.05, * * p<0.01, * * * p<0.001$ when compared with RA-no ILD at baseline, Mann-Whitney U test $p$ values are presented. 
Table 2 Baseline parameters and disease outcome at Year 5

\begin{tabular}{|c|c|c|}
\hline \multirow[b]{2}{*}{ Baseline parameters } & \multicolumn{2}{|c|}{ Outcome at Year 5} \\
\hline & $\begin{array}{l}\text { Progression } \\
\text { (Worse) } \\
(n=19)\end{array}$ & $\begin{array}{c}\text { No-progression } \\
\text { (Unchanged/Stable+Improved) } \\
(\mathbf{n}=\mathbf{4 1})\end{array}$ \\
\hline \multicolumn{3}{|l|}{ Demographic parameters } \\
\hline \multicolumn{3}{|l|}{ ILA score } \\
\hline ILA score 0 & 3 & 19 \\
\hline ILA score 1 & 8 & 15 \\
\hline ILA score 2 & 3 & 7 \\
\hline ILA score 3 & 5 & 0 \\
\hline Age, years, mean \pm SD & $61.1 \pm 16.0^{* *}$ & $45.1 \pm 16.1$ \\
\hline Female, no. $(\%)$ & $13(68)$ & $35(85)$ \\
\hline Pack-years of smoking, mean \pm SD & $3.7 \pm 9.0$ & $1.6 \pm 7.3$ \\
\hline Pack-years of secondhand smoking, mean $\pm \mathrm{SD}$ & $2.1 \pm 9.2$ & $1.8 \pm 5.2$ \\
\hline \multicolumn{3}{|l|}{ RA parameters, median (IQR) } \\
\hline $\mathrm{RF}, \mathrm{IU} / \mathrm{ml}$ & $195.0(113.0-697.0)^{*}$ & $105.0(26.7-310.5)$ \\
\hline Anti-CCP, units/ml & $255.0(53.2-500.0)$ & $274.8(60.2-500.0)$ \\
\hline DAS28 & $4.2(3.2-5.2)$ & $4.1(2.9-5.3)$ \\
\hline Duration of RA, years & $3.0(1.0-10.0)$ & $2.0(1.0-9.5)$ \\
\hline \multicolumn{3}{|l|}{$\begin{array}{l}\text { Cumulative dosages of medications used over } \\
\text { 5-year(mg), median (IQR) }\end{array}$} \\
\hline Methotrexate & $260.0(52.0-520)^{*}$ & $325.0(211.3-390.0)$ \\
\hline Leflunomide & $0(0.0-1825.0)$ & $0(0.0-1825.0)$ \\
\hline Corticosteroid & $0(0-0)$ & $0(0-0)$ \\
\hline \multicolumn{3}{|l|}{ Respiratory parameters, no. (\%) } \\
\hline Cough & $4(21)^{* *}$ & $0(0)$ \\
\hline Dyspnea & $4(21)^{* *}$ & $0(0)$ \\
\hline \multicolumn{3}{|l|}{ Spirometric parameters, median (IQR) } \\
\hline FEV1, percent of predicted & $79.0(59.8-89.8)$ & $82.0(75.8-93.8)$ \\
\hline FVC, percent of predicted & $75.5(62.0-86.0)$ & $79.0(75.0-93.0)$ \\
\hline DLco, percent of predicted & $58.0(51.0-72.0)^{* *}$ & $86.0(68.8-102.0)$ \\
\hline \multicolumn{3}{|l|}{ HRCT abnormalities, no. (\%) } \\
\hline UIP pattern & $5(26)$ & $0(0)$ \\
\hline Non-UIP pattern & $14(74)^{* *}$ & $41(100)$ \\
\hline \multicolumn{3}{|l|}{$\begin{array}{l}\text { Quantitative Modified ILD scoring in HRCT, } \\
\text { median (IQR) }\end{array}$} \\
\hline & $2.0(1.0-12.0)^{*}$ & $1.5(0.0-3.5)$ \\
\hline
\end{tabular}


RA-ILD outcomes at Year 5 were defined as progression versus no-progression based on changes of the Quantitative Modified ILD HRCT scores from Year 0 to Year 5. Unchanged/stable $(\leq 10 \%$ change) and improved ( $\geq 10 \%$ decrease) in the Quantitative Modified ILD scores were designated as no-progression, while worsening scores ( $>10 \%$ increase in Quantitative Modified ILD scores) were classified as progression. Among the 56 patients who were alive at Year 5, 39 had available PFT data from both Year 0 and Year 5 time points. Baseline age, RF, cough, dyspnea, DLco\% and non-UIP HRCT pattern in the progression group were significantly different compared to the no-progression group $(p<0.01,0.05,0.01,0.01,0.01,0.01$ respectively). The cumulative dosage of methotrexate $(\mathrm{mg})$ used over 5-years in the progression group was significantly lower than in the no-progression group $(p=0.027)$. ${ }^{*} p<0.05, * * p<0.01, * * * p<0.001$, progression group versus non-progression group respectively. p-values were derived from Mann-Whitney rank sum analysis. 
Table 3 Factors predicting radiographic progression in RA-ILD

\begin{tabular}{|c|c|c|c|}
\hline & Progression $(n=19)$ & No-progression $(n=41)$ & $p$-value \\
\hline Age, years & $61.1 \pm 16.0$ & $45.1 \pm 16.1$ & 0.002 \\
\hline $\mathrm{RF}, \mathrm{IU} / \mathrm{ml}$ & $195.0(113.0-697.0)$ & $105.0(26.7-310.5)$ & 0.044 \\
\hline Anti-CCP, untis/ml & $255.0(53.2-500.0)$ & $274.8(60.2-500.0)$ & 0.898 \\
\hline DAS28 & $4.2(3.2-5.2)$ & $4.1(2.9-5.3)$ & 0.987 \\
\hline Duration of RA, years & $3.0(1.0-10.0)$ & $2.0(1.0-9.5)$ & 0.537 \\
\hline CXCL9/MIG $(\log$ Year $5-\log$ Year 0) & $-0.1(-0.8-0.3)$ & $-0.3(-0.7-0.0)$ & 0.215 \\
\hline CXCL10/IP10 (log Year $5-\log$ Year 0) & $-0.8[-1.3-0.0]$ & $-0.6[-0.9-(-0.3)]$ & 0.667 \\
\hline CXCL11/I-TAC (log Year $5-\log$ Year 0) & $0.0[-0.9-0.4]$ & $0.0(-1.5-0.0)$ & 0.036 \\
\hline MMP13 (log Year 5 - log Year 0) & $0.0(-0.4-0.6)$ & $-0.4(-0.8-0.0)$ & 0.014 \\
\hline
\end{tabular}

After normality testing (K-S test) for the nine indices, age conformed to the normal distribution. The actual values were presented as mean \pm standard deviation. RF,

anti-CCP, DSA28 and disease duration at baseline did not adhere to a normal distribution and were therefore converted by logarithm transformation and presented as median

(interquartile range). Changes in cytokine levels of CXCL11/I-TAC and MMP-13 from Year 0 to Year 5 were converted by logarithm transformation and expressed as (log

Year 5 - log Year 0). Significant differences were found between the progression and the no-progression groups in age, RF, CXCL11/I-TAC (log Year 5 - log Year 0) and MMP13 (log Year 5 - $\log$ Year 0). Other variables did not show statistically significant differences.

$* p<0.05, * * p<0.01, * * * p<0.001$ 
Relationship between selected risk factors and progression of RA-ILD

\begin{tabular}{lcc}
\hline & beta coefficient & $P$-value \\
\hline Age & 0.054 & 0.017 \\
MMP13 (log Year 5 - log Year 0) & 1.372 & 0.026 \\
Constant & -3.527 & 0.004 \\
\hline
\end{tabular}

Descriptive analyses showed differences in risk of RA-ILD progression for age, RF, and changes (log Year 5-log Year 0) in serum levels of CXCL11/I- and MMP13. We conducted Spearman correlation analyses for continuous variables and selected one member of each pair of correlated variables ( $r>0.3$ and $p<0.05)$ to include into the logistic regression model for avoiding multicollinearity. With those steps, age and MMP-13 (log Year 5-log Year 0) were selected to include in the final model. 


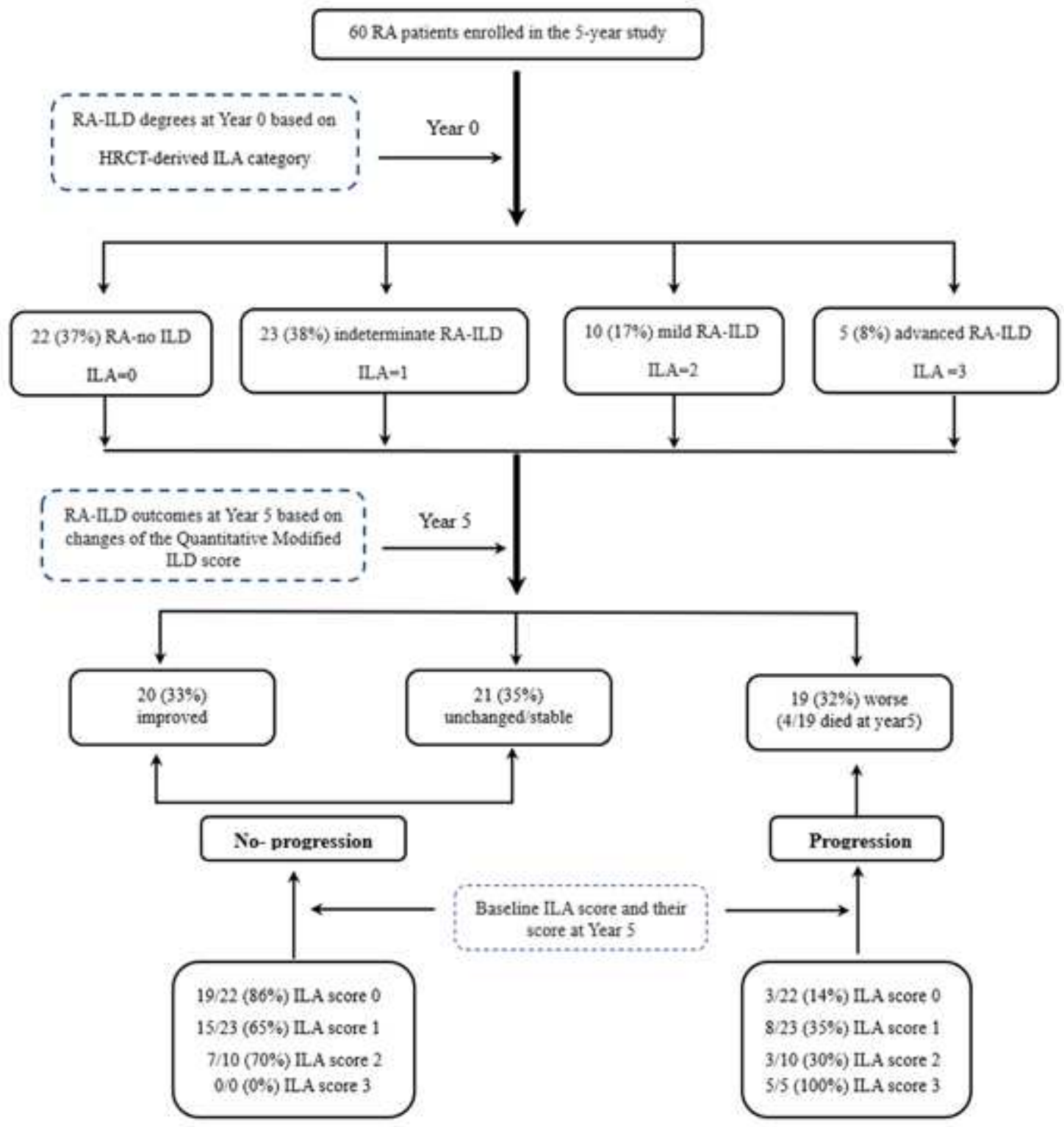

\section{Figure 1}

Study enrollment at Year 0 and Year 5 A flow diagram of the study shows the different degrees of RA-ILD based on 0-3 ILA scores in HRCT at Year 0 and outcome at Year 5 based on changes of Quantitative Modified ILD scores. Changes in the Quantitative Modified ILD score between 0-10\% at Year 0 and Year 5 were classified as stable, while reduction of ILD scores by greater than $10 \%$ were classified as better (score reduction $>10 \%$ ) and changes more than $10 \%$ increased as worse. HRCT = high-resolution computed tomography; ILD = interstitial lung disease; ILA= interstitial lung abnormality; RA=rheumatoid arthritis. 

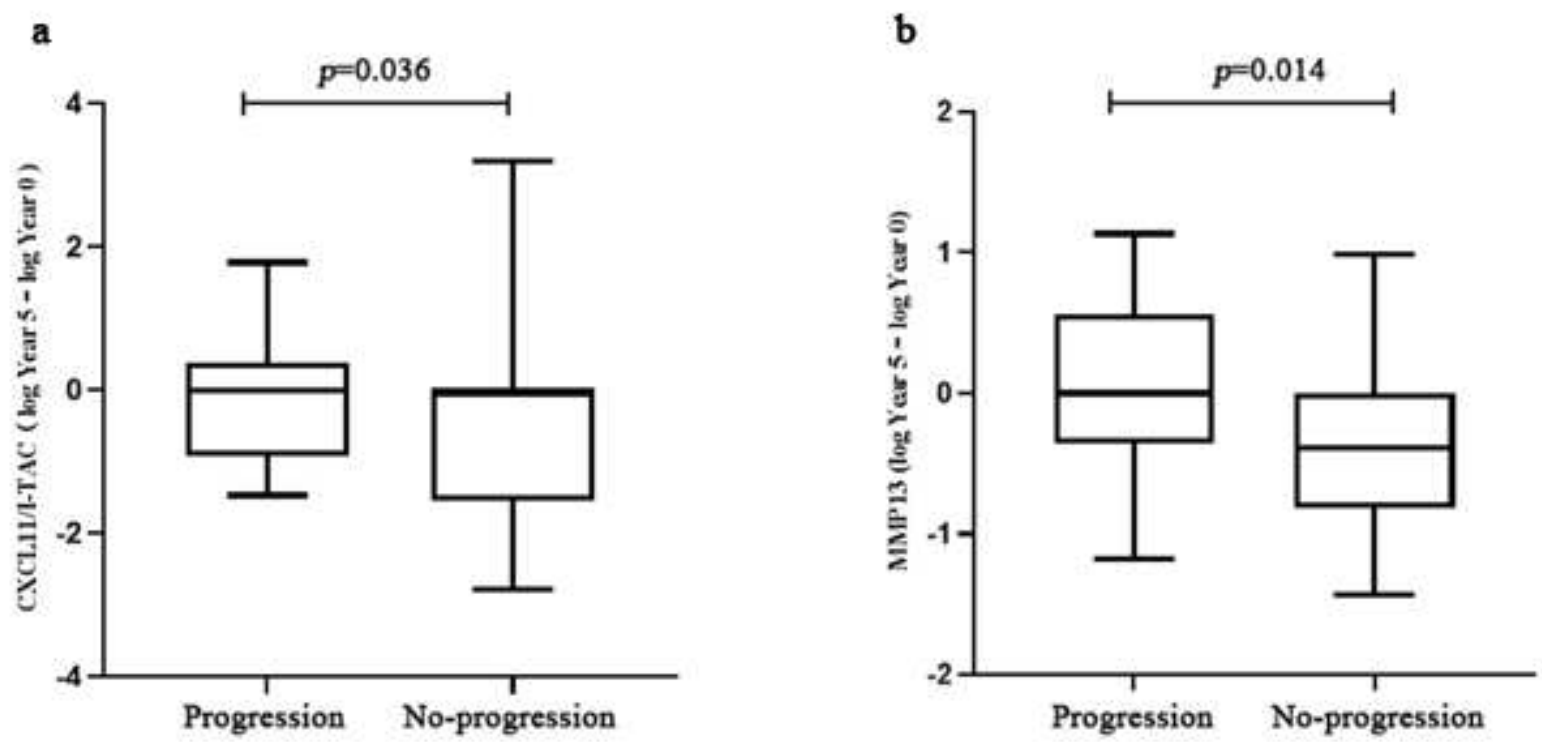

\section{Figure 2}

Relationship between changes in serum levels of biomarkers from Year 0 to Year 5 and radiographic outcome. Changes of serum levels of A) CXCL11/I-TAC and B) MMP-13 from Year 0 to Year 5 (log Year 5 $\log$ Year 0 ) ( $y$-axis) were significantly associated with RA-ILD outcomes (progression versus noprogression, $x$-axis) assessed by changes in Quantitative Modified ILD scores ( $p=0.036, p=0.014$, respectively). Horizontal bars signify median values. 


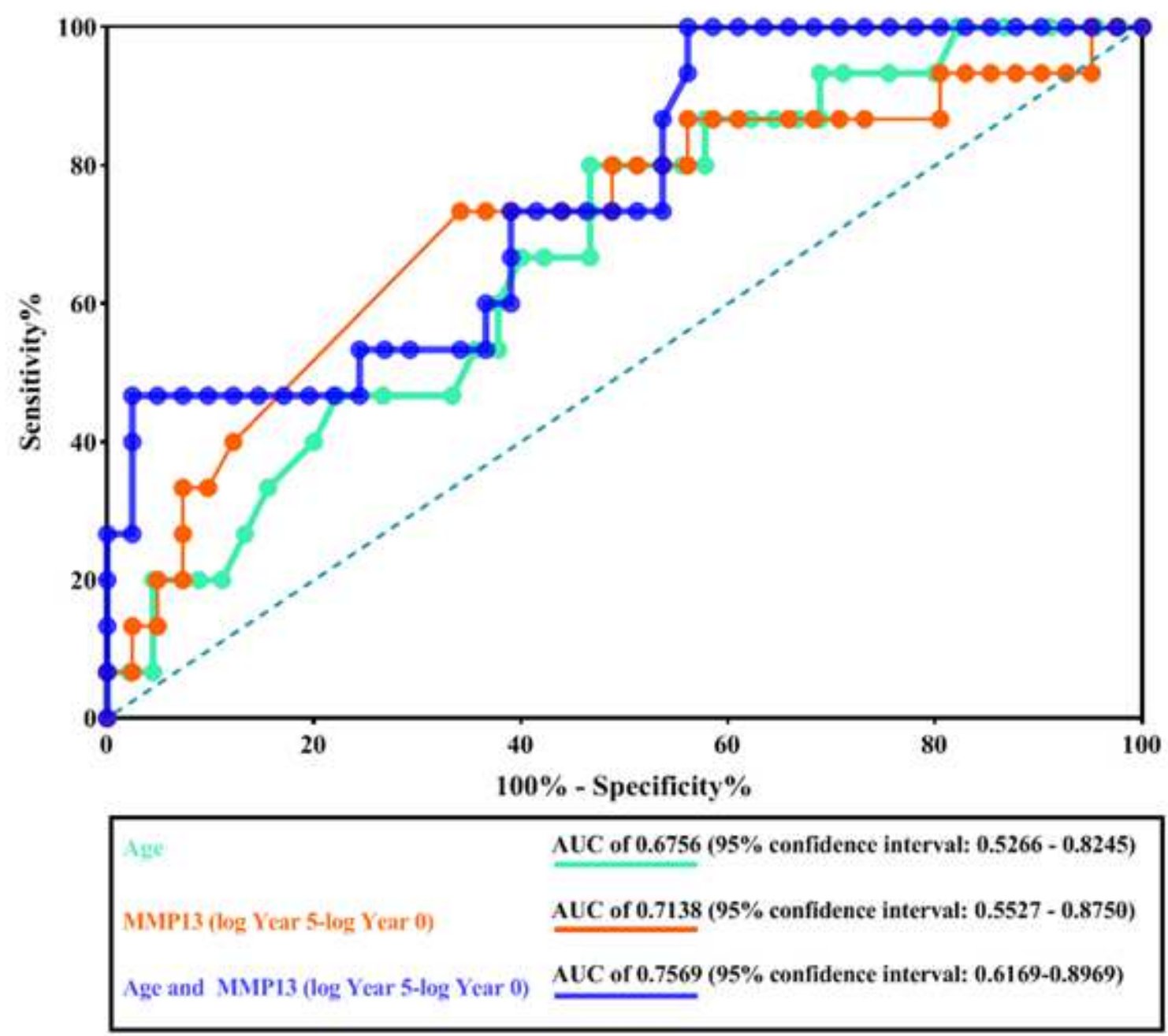

Figure 3

ROC analyses of the disease prediction models Age and changes in serum levels of MMP13 $ه$ log Year 5log Year O囚over 5-years were fit into the final logistic regression model. ROC analyses for the models: 1) with age; 2) with 27 changes in MMP13 level; and 3) with the combination of age and changes in MMP13 level demonstrated the model with the combinations of the variables predicts the risk of progression in RA-ILD (as measured by Quantitative Modified ILD scores) the best. The respective AUCs for those models are $0.6756,0.7138$ and $0.7569(p=0.04,0.02$ and $<0.01)$.

\section{Supplementary Files}

This is a list of supplementary files associated with this preprint. Click to download.

- Supplementary105.pdf 